\title{
A New Approach for Solving Fractional Partial Differential Equations
}

\author{
Fanwei Meng \\ School of Mathematical Sciences, Qufu Normal University, Qufu 273165, China \\ Correspondence should be addressed to Fanwei Meng; fengqinghua1978@126.com
}

Received 20 January 2013; Accepted 18 April 2013

Academic Editor: Livija Cveticanin

Copyright (C) 2013 Fanwei Meng. This is an open access article distributed under the Creative Commons Attribution License, which permits unrestricted use, distribution, and reproduction in any medium, provided the original work is properly cited.

\begin{abstract}
We propose a new approach for solving fractional partial differential equations based on a nonlinear fractional complex transformation and the general Riccati equation and apply it to solve the nonlinear time fractional biological population model and the (4+1)-dimensional space-time fractional Fokas equation. As a result, some new exact solutions for them are obtained. This approach can be suitable for solving fractional partial differential equations with more general forms than the method proposed by S. Zhang and H.-Q. Zhang (2011).
\end{abstract}

\section{Introduction}

Fractional differential equations are generalizations of classical differential equations of integer order. In recent decades, fractional differential equations have been the focus of many studies due to their frequent appearance in various applications in physics, biology, engineering, signal processing, systems identification, control theory, finance, and fractional dynamics. Many articles have investigated some aspects of fractional differential equations, such as the existence and uniqueness of solutions to the Cauchy-type problems, the methods for explicit and numerical solutions, and the stability of solutions [1-8]. Among the investigations for fractional differential equations, the research for seeking exact solutions and numerical solutions of fractional differential equations is an important topic. Many powerful and efficient methods have been proposed to obtain numerical solutions and exact solutions of fractional differential equations so far. For example, these methods include the Adomian decomposition method $[9,10]$, the variational iterative method [11-13], the homotopy perturbation method $[14,15]$, the differential transformation method [16], the finite difference method [17], the finite element method [18], and the fractional Riccati subequation method $[19,20]$. Based on these methods, a variety of fractional differential equations have been investigated.

Recently, S. Zhang and H.-Q. Zhang [21] proposed a new direct algebraic method named fractional subequation method for solving fractional partial differential equations based on the homogeneous balance principle, the modified Riemann-Liouville derivative by Jumarie [22], and the fractional Riccati equation. The main idea of this method lies in that by a linear transformation $\xi=\xi\left(t, x_{1}, x_{2}, \ldots, x_{n}\right)$, a given fractional partial differential equation expressed in independent variables $t, x_{1}, x_{2}, \ldots, x_{n}$, in which the highestorder derivative and nonlinear term are involved, is turned into another fractional ordinary differential equation, whose solutions are supposed to have the form $u(\xi)=\sum_{i=0}^{n} a_{i} \phi^{i}$, where $\phi=\phi(\xi)$ satisfies the fractional Riccati equation $D_{\xi}^{\alpha} \phi=$ $\sigma+\phi^{2}$. Based on this concept, the authors established successfully some exact solutions for the nonlinear time fractional biological population model and the $(4+1)$ dimensional space-time fractional Fokas equation. We note that as long as a different nonlinear fractional complex transformation form is taken for $\xi$, then a certain fractional partial differential equation can be turned into another ordinary differential equation of integer order, which permits the use of the Riccati equation of integer order $\phi^{\prime}=\sigma+\phi^{2}$ instead of the fractional Riccati equation in the analysis of [21]. Subsequently, new exact solutions for the two equations can be obtained. Furthermore, we notice that the method in [21] is only suitable for solving those fractional partial differential equations involving fractional partial derivatives of certain orders, for example, 
$D_{t}^{\alpha} u, D_{x}^{\alpha} u, D_{y}^{\alpha} u, D_{t}^{2 \alpha} u, D_{x}^{2 \alpha} u, D_{y}^{2 \alpha} u, \ldots$, which implies that all of the orders are integer multiples of $\alpha$. For some fractional partial differential equations involving fractional partial derivatives of arbitrarily different orders with respect to different variables, for example, $D_{t}^{\alpha} u, D_{x}^{\beta} u, D_{y}^{\gamma} u$, $D_{t}^{2 \alpha} u, D_{x}^{2 \beta} u, D_{y}^{2 \gamma} u, \ldots$, or some involving either fractional partial derivatives or integer order derivatives, for example, $D_{t}^{\alpha} u, u_{x}^{\prime}, D_{y}^{\gamma} u, D_{t}^{2 \alpha} u, u_{x}^{\prime \prime}, D_{y}^{2 \gamma} u, \ldots$, the method in [21] is invalid.

The object of this paper is to propose a different way from [21] based on the Riccati equation of integer order and a nonlinear fractional complex transformation $\xi=$ $\xi\left(t, x_{1}, x_{2}, \ldots, x_{n}\right)$ for solving fractional partial differential equations. The proposed approach will still be valid in the last two cases mentioned above.

\section{The Jumarie Modified Riemann-Liouville Derivative and Description of the Proposed Method}

The Jumarie modified Riemann-Liouville derivative of order $\alpha$ is defined by the following expression [22]:

$$
\begin{aligned}
D_{x}^{\alpha} f(x) & =\lim _{h \rightarrow 0} \frac{\Delta_{\alpha} f(x)}{h^{\alpha}} \\
& =\lim _{h \rightarrow 0} \frac{\sum_{k=0}^{\infty}(-1)^{k}\left(\begin{array}{l}
\alpha \\
k
\end{array}\right) f(x+(\alpha-k) h)}{h^{\alpha}} .
\end{aligned}
$$

We list some important properties for the modified Riemann-Liouville derivative as follows [19-21]:

$$
\begin{gathered}
D_{t}^{\alpha} t^{r}=\frac{\Gamma(1+r)}{\Gamma(1+r-\alpha)} t^{r-\alpha} \\
D_{t}^{\alpha}(f(t) g(t))=g(t) D_{t}^{\alpha} f(t)+f(t) D_{t}^{\alpha} g(t), \\
D_{t}^{\alpha} f[g(t)]=f_{g}^{\prime}[g(t)] D_{t}^{\alpha} g(t)=D_{g}^{\alpha} f[g(t)]\left(g^{\prime}(t)\right)^{\alpha} .
\end{gathered}
$$

In the following, we give the description of our method.

Suppose that a fractional partial differential equation, say in the independent variables $t, x_{1}, x_{2}, \ldots, x_{n}$, is given by

$$
\begin{gathered}
P\left(u, u_{t}, u_{x_{1}}, \ldots, u_{x_{n}}, D_{t}^{\alpha} u, D_{x_{1}}^{\beta} u, \ldots, D_{x_{n}}^{\gamma} u\right. \\
D_{t}^{2 \alpha} u, D_{x_{1}}^{2 \beta} u, \ldots, D_{x_{n}}^{2 \gamma} u, D_{t, x_{1}}^{\alpha+\beta} u, \ldots, \\
\left.D_{t, x_{n}}^{\alpha+\gamma} u, \ldots, D_{x_{1}, x_{n}}^{\beta+\gamma} u, \ldots\right)=0
\end{gathered}
$$

where $u=u\left(t, x_{1}, x_{2}, \ldots, x_{n}\right)$ is an unknown function and $P$ is a polynomial in $u$ and its various partial derivatives, in which the highest-order derivative and nonlinear term are involved.

Step 1. Suppose that

$$
u\left(t, x_{1}, x_{2}, \ldots, x_{n}\right)=U(\xi), \quad \xi=\xi\left(t, x_{1}, x_{2}, \ldots, x_{n}\right),
$$

such that (5) can be turned into the following ordinary differential equation of integer order with respect to the variable $\xi$ :

$$
\widetilde{P}\left(U, U^{\prime}, U^{\prime \prime}, \ldots\right)=0 \text {. }
$$

Step 2. Suppose that the solution of (7) can be expressed by a polynomial in $\phi$ as follows:

$$
U(\xi)=\sum_{i=0}^{n} a_{i} \phi^{i},
$$

where $\phi=\phi(\xi)$ satisfies the Riccati equation

$$
\phi^{\prime}=\sigma+\phi^{2}
$$

and $a_{i}, i=0,1, \ldots, n$ are all constants to be determined later. The positive integer $n$ can be determined by considering the homogeneous balance between the highest-order derivative and nonlinear term appearing in (7).

We list the exact solutions of (9) as follows, which is known to us as

$$
\phi(\xi)= \begin{cases}-\sqrt{-\sigma} \tanh (\sqrt{-\sigma} \xi), & \sigma<0, \\ -\sqrt{-\sigma} \operatorname{coth}(\sqrt{-\sigma} \xi), & \sigma<0, \\ \sqrt{\sigma} \tan (\sqrt{\sigma} \xi), & \sigma>0, \\ -\sqrt{\sigma} \cot (\sqrt{\sigma} \xi), & \sigma>0, \\ -\frac{1}{\xi+\omega}, & \omega \text { is a constant, } \sigma=0 .\end{cases}
$$

Step 3. Substituting (8) into (7) and using (9), the left-hand side of (7) is converted to another polynomial in $\phi^{j}$ after eliminating the denominator. Equating each coefficient of this polynomial to zero yields a set of algebraic equations for $a_{i}, i=0,1, \ldots, n$.

Step 4. Solving the equations system in Step 3, and by using the solutions of (9), we can construct a variety of exact solutions of (5).

\section{Applications of the Proposed Method}

In this section, we will apply the described method in Section 2 to the nonlinear time fractional biological population model and the (4+1)-dimensional space-time fractional Fokas equation.

3.1. Nonlinear Time Fractional Biological Population Model. We consider the nonlinear time fractional biological population model:

$$
D_{t}^{\alpha} u=\left(u^{2}\right)_{x x}+\left(u^{2}\right)_{y y}+h\left(u^{2}-r\right),
$$

where $h, r$ are constants.

Suppose that $u(x, y, t)=U(\xi)$, where $\xi=k x+i k y+\left(c t^{\alpha} /\right.$ $\Gamma(1+\alpha))+\xi_{0}, k, c, \xi_{0}$ are all constants with $k, c \neq 0$, and $i$ is the unit of imaginary numbers. Then, by the use of (4), equation (18) can be turned into

$$
c U^{\prime}=h\left(U^{2}-r\right) .
$$


Suppose that the solution of (12) can be expressed by

$$
U(\xi)=\sum_{i=0}^{n} a_{i} \phi^{i},
$$

where $\phi=\phi(\xi)$ satisfies (9). By balancing the order between the highest-order derivative term and nonlinear term in (12), we can obtain $n=1$. So, we have

$$
U(\xi)=a_{0}+a_{1} \phi
$$

Substituting (14) into (12) and collecting all the terms with the same power of $\phi$ together, equating each coefficient to zero yields a set of algebraic equations. Solving these equations yields

$$
a_{0}=0, \quad a_{1}=\frac{c}{h}, \quad \sigma=-h^{2} r c^{-2} .
$$

Substituting the result above into (14) and combining it with (10), we can obtain the following exact solutions to (11):

$$
\begin{aligned}
& u_{1}(x, y, t) \\
& =-\sqrt{r} \tanh \left[h c^{-1} \sqrt{r}\left(k x+i k y+\frac{c t^{\alpha}}{\Gamma(1+\alpha)}+\xi_{0}\right)\right], \\
& r>0 \text {, } \\
& u_{2}(x, y, t) \\
& =-\sqrt{r} \operatorname{coth}\left[h c^{-1} \sqrt{r}\left(k x+i k y+\frac{c t^{\alpha}}{\Gamma(1+\alpha)}+\xi_{0}\right)\right], \\
& r>0 \text {, } \\
& u_{3}(x, y, t) \\
& =\sqrt{-r} \tan \left[h c^{-1} \sqrt{-r}\left(k x+i k y+\frac{c t^{\alpha}}{\Gamma(1+\alpha)}+\xi_{0}\right)\right], \\
& r<0 \text {, } \\
& u_{4}(x, y, t) \\
& =-\sqrt{-r} \cot \left[h c^{-1} \sqrt{-r}\left(k x+i k y+\frac{c t^{\alpha}}{\Gamma(1+\alpha)}+\xi_{0}\right)\right], \\
& r<0, \\
& \begin{array}{r}
u_{5}(x, y, t) \\
=\frac{-c}{h\left(k x+i k y+\left(c t^{\alpha} / \Gamma(1+\alpha)\right)+\xi_{0}+\omega\right)}, \\
r=0,
\end{array}
\end{aligned}
$$

where $\xi_{0} k, c$ are all arbitrary constants.

Remark 1. As one can see, the obtained solutions in (16) for the nonlinear time fractional biological population model are different from the results in [21] and are new exact solutions so far to our best knowledge.
3.2. The Space-Time Fractional Fokas Equation. We consider the space-time fractional Fokas equation

$$
\begin{aligned}
& 4 \frac{\partial^{2 \alpha} q}{\partial t^{\alpha} \partial x_{1}^{\alpha}}-\frac{\partial^{4 \alpha} q}{\partial x_{1}^{3 \alpha} \partial x_{2}^{\alpha}}+\frac{\partial^{4 \alpha} q}{\partial x_{2}^{3 \alpha} \partial x_{1}^{\alpha}}+12 \frac{\partial^{\alpha} q}{\partial x_{1}^{\alpha}} \frac{\partial^{\alpha} q}{\partial x_{2}^{\alpha}} \\
& +12 q \frac{\partial^{2 \alpha} q}{\partial x_{1}^{\alpha} \partial x_{2}^{\alpha}}-6 \frac{\partial^{2 \alpha} q}{\partial y_{1}^{\alpha} \partial y_{2}^{\alpha}}=0, \quad 0<\alpha \leq 1 .
\end{aligned}
$$

Suppose that $q\left(t, x_{1}, x_{2}, y_{1}, y_{2}\right)=U(\xi)$, where $\xi=\left(k_{1} x_{1}^{\alpha} /\right.$ $\Gamma(1+\alpha))+\left(k_{2} x_{2}^{\alpha} / \Gamma(1+\alpha)\right)+\left(l_{1} y_{1}^{\alpha} / \Gamma(1+\alpha)\right)+\left(l_{2} y_{2}^{\alpha} / \Gamma(1+\right.$ $\alpha))+\left(c t^{\alpha} / \Gamma(1+\alpha)\right)+\xi_{0}, k_{1}, k_{2}, l_{1} l_{2}, c, \xi_{0}$ are all constants with $k_{1}, k_{2}, l_{1}, l_{2}, c \neq 0$. Then, by the use of (4), equation (17) can be turned into

$$
\begin{aligned}
4 c k_{1} U^{\prime \prime}- & k_{1}^{3} k_{2} U^{(4)}+k_{2}^{3} k_{1} U^{(4)} \\
& +12 k_{1} k_{2} U^{\prime 2}+12 k_{1} k_{2} U U^{\prime \prime}-6 l_{1} l_{2} U^{\prime \prime}=0 .
\end{aligned}
$$

Suppose that the solution of (18) can be expressed by

$$
U(\xi)=\sum_{i=0}^{n} a_{i} \phi^{i},
$$

where $\phi=\phi(\xi)$ satisfies (9). By balancing the order between the highest-order derivative term and nonlinear term in (18), we can obtain $n=2$. So, we have

$$
U(\xi)=a_{0}+a_{1} \phi+a_{2} \phi^{2} .
$$

Substituting (20) into (18) and collecting all the terms with the same power of $\phi$ together, equating each coefficient to zero yields a set of algebraic equations. Solving these equations yields

$$
\begin{gathered}
a_{0}=\frac{4 k_{1}^{3} k_{2} \sigma-4 k_{1} k_{2}^{3} \sigma-2 c k_{1}+3 l_{1} l_{2}}{6 k_{1} k_{2}}, \\
a_{1}=0, \quad a_{2}=k_{1}^{2}-k_{2}^{2} .
\end{gathered}
$$

Substituting the result above into (19) and combining it with (10), we can obtain the following exact solutions to (17):

$$
\begin{aligned}
& q_{1}\left(t, x_{1}, x_{2}, y_{1}, y_{2}\right) \\
& =\frac{4 k_{1}^{3} k_{2} \sigma-4 k_{1} k_{2}^{3} \sigma-2 c k_{1}+3 l_{1} l_{2}}{6 k_{1} k_{2}}-\left(k_{1}^{2}-k_{2}^{2}\right) \\
& \quad \times \sigma \tanh ^{2}\left[\sqrt { - \sigma } \left(\frac{k_{1} x_{1}^{\alpha}}{\Gamma(1+\alpha)}+\frac{k_{2} x_{2}^{\alpha}}{\Gamma(1+\alpha)}+\frac{l_{1} y_{1}^{\alpha}}{\Gamma(1+\alpha)}\right.\right. \\
& \left.\left.\quad+\frac{l_{2} y_{2}^{\alpha}}{\Gamma(1+\alpha)}+\frac{c t^{\alpha}}{\Gamma(1+\alpha)}+\xi_{0}\right)\right], \\
& q_{2}\left(t, x_{1}, x_{2}, y_{1}, y_{2}\right) \quad \\
& =\frac{4 k_{1}^{3} k_{2} \sigma-4 k_{1} k_{2}^{3} \sigma-2 c k_{1}+3 l_{1} l_{2}}{6 k_{1} k_{2}}-\left(k_{1}^{2}-k_{2}^{2}\right) \\
& \quad \times \sigma \operatorname{coth}^{2}\left[\sqrt { - \sigma } \left(\frac{k_{1} x_{1}^{\alpha}}{\Gamma(1+\alpha)}+\frac{k_{2} x_{2}^{\alpha}}{\Gamma(1+\alpha)}+\frac{l_{1} y_{1}^{\alpha}}{\Gamma(1+\alpha)}\right.\right.
\end{aligned}
$$




$$
\begin{aligned}
& \left.\left.+\frac{l_{2} y_{2}^{\alpha}}{\Gamma(1+\alpha)}+\frac{c t^{\alpha}}{\Gamma(1+\alpha)}+\xi_{0}\right)\right] \\
& \sigma<0, \\
& q_{3}\left(t, x_{1}, x_{2}, y_{1}, y_{2}\right) \\
& =\frac{4 k_{1}^{3} k_{2} \sigma-4 k_{1} k_{2}^{3} \sigma-2 c k_{1}+3 l_{1} l_{2}}{6 k_{1} k_{2}}+\left(k_{1}^{2}-k_{2}^{2}\right) \\
& \times \sigma \tan ^{2}\left[\sqrt { \sigma } \left(\frac{k_{1} x_{1}^{\alpha}}{\Gamma(1+\alpha)}+\frac{k_{2} x_{2}^{\alpha}}{\Gamma(1+\alpha)}+\frac{l_{1} y_{1}^{\alpha}}{\Gamma(1+\alpha)}\right.\right. \\
& \left.\left.+\frac{l_{2} y_{2}^{\alpha}}{\Gamma(1+\alpha)}+\frac{c t^{\alpha}}{\Gamma(1+\alpha)}+\xi_{0}\right)\right], \\
& \sigma>0 \\
& q_{4}\left(t, x_{1}, x_{2}, y_{1}, y_{2}\right) \\
& =\frac{4 k_{1}^{3} k_{2} \sigma-4 k_{1} k_{2}^{3} \sigma-2 c k_{1}+3 l_{1} l_{2}}{6 k_{1} k_{2}}-\left(k_{1}^{2}-k_{2}^{2}\right) \\
& \times \sigma \cot ^{2}\left[\sqrt { \sigma } \left(\frac{k_{1} x_{1}^{\alpha}}{\Gamma(1+\alpha)}+\frac{k_{2} x_{2}^{\alpha}}{\Gamma(1+\alpha)}+\frac{l_{1} y_{1}^{\alpha}}{\Gamma(1+\alpha)}\right.\right. \\
& \left.\left.+\frac{l_{2} y_{2}^{\alpha}}{\Gamma(1+\alpha)}+\frac{c t^{\alpha}}{\Gamma(1+\alpha)}+\xi_{0}\right)\right], \\
& \sigma>0 \\
& q_{5}\left(t, x_{1}, x_{2}, y_{1}, y_{2}\right) \\
& \begin{aligned}
&= \frac{4 k_{1}^{3} k_{2} \sigma-4 k_{1} k_{2}^{3} \sigma-2 c k_{1}+3 l_{1} l_{2}}{6 k_{1} k_{2}} \\
&+\frac{\left(k_{1}^{2}-k_{2}^{2}\right) \Gamma(1+\alpha)}{k_{1} x_{1}^{\alpha}+k_{2} x_{2}^{\alpha}+l_{1} y_{1}^{\alpha}+l_{2} y_{2}^{\alpha}+c t^{\alpha}+\Gamma(1+\alpha)\left(\xi_{0}+\omega\right)}, \\
& \sigma=0,
\end{aligned}
\end{aligned}
$$

where $\xi_{0}, k_{1}, k_{2}, l_{1}, l_{2}, c, \omega$ are all arbitrary constants.

Remark 2. The established solutions in (22) for the $(4+1)$ dimensional space-time fractional Fokas equation are different from the results in [21] and also have not been reported by other authors in the literature.

Remark 3. It is worth to notice that if (17) is replaced by the following equation:

$$
\begin{aligned}
& 4 \frac{\partial^{\alpha+\beta} q}{\partial t^{\alpha} \partial x_{1}^{\beta}}-\frac{\partial^{3 \beta+\alpha} q}{\partial x_{1}^{3 \beta} \partial x_{2}^{\alpha}}+\frac{\partial^{3 \alpha+\beta} q}{\partial x_{2}^{3 \alpha} \partial x_{1}^{\beta}}+12 \frac{\partial^{\beta} q}{\partial x_{1}^{\beta}} \frac{\partial^{\alpha} q}{\partial x_{2}^{\alpha}} \\
& +12 q \frac{\partial^{\beta+\alpha} q}{\partial x_{1}^{\beta} \partial x_{2}^{\alpha}}-6 \frac{\partial^{1+\gamma} q}{\partial y_{1} \partial y_{2}^{\gamma}}=0, \quad 0<\alpha, \beta, \gamma \leq 1,
\end{aligned}
$$

then one can see that the fractional subequation method in [1] is invalid here, while we can still reduce (23) to (18) if we take the transformation $\xi=\left(k_{1} x_{1}^{\beta} / \Gamma(1+\beta)\right)+\left(k_{2} x_{2}^{\alpha} / \Gamma(1+\right.$ $\alpha))+l_{1} y_{1}+\left(l_{2} y_{2}^{\gamma} / \Gamma(1+\gamma)\right)+\left(c t^{\alpha} / \Gamma(1+\alpha)\right)+\xi_{0}$. So, the method proposed here can be suitable for more general cases.

\section{Conclusions}

We have proposed a new subequation method for fractional partial differential equations based on the Riccati equation of integer order and a nonlinear fractional complex transformation and applied it to the nonlinear time fractional biological population model and the (4+1)-dimensional spacetime fractional Fokas equation. As a result, some new exact solutions for them are established. As one can see, this method has more general applications than the fractional subequation method and can be applied to other fractional partial differential equations.

\section{Acknowledgments}

This work is partially supported by the National Natural Science Foundation of China (11171178) and the Program for Scientific Research Innovation Team in Colleges and Universities of Shandong Province. The author would like to thank the reviewers very much for their valuable suggestions on the paper.

\section{References}

[1] A. Saadatmandi and M. Dehghan, "A new operational matrix for solving fractional-order differential equations," Computers \& Mathematics with Applications, vol. 59, no. 3, pp. 1326-1336, 2010.

[2] Y. Zhou, F. Jiao, and J. Li, "Existence and uniqueness for $p$-type fractional neutral differential equations," Nonlinear Analysis: Theory, Methods \& Applications A, vol. 71, no. 7-8, pp. 27242733, 2009.

[3] L. Galeone and R. Garrappa, "Explicit methods for fractional differential equations and their stability properties," Journal of Computational and Applied Mathematics, vol. 228, no. 2, pp. 548-560, 2009.

[4] J. C. Trigeassou, N. Maamri, J. Sabatier, and A. Oustaloup, "A Lyapunov approach to the stability of fractional differential equations," Signal Processing, vol. 91, no. 3, pp. 437-445, 2011.

[5] W. Deng, "Smoothness and stability of the solutions for nonlinear fractional differential equations," Nonlinear Analysis: Theory, Methods \& Applications A, vol. 72, no. 3-4, pp. 1768-1777, 2010.

[6] F. Ghoreishi and S. Yazdani, "An extension of the spectral Tau method for numerical solution of multi-order fractional differential equations with convergence analysis," Computers \& Mathematics with Applications, vol. 61, no. 1, pp. 30-43, 2011.

[7] J. T. Edwards, N. J. Ford, and A. C. Simpson, "The numerical solution of linear multi-term fractional differential equations: systems of equations," Journal of Computational and Applied Mathematics, vol. 148, no. 2, pp. 401-418, 2002.

[8] M. Muslim, "Existence and approximation of solutions to fractional differential equations," Mathematical and Computer Modelling, vol. 49, no. 5-6, pp. 1164-1172, 2009.

[9] A. M. A. El-Sayed and M. Gaber, "The Adomian decomposition method for solving partial differential equations of fractal order in finite domains," Physics Letters A, vol. 359, no. 3, pp. 175-182, 2006.

[10] A. M. A. El-Sayed, S. H. Behiry, and W. E. Raslan, "Adomian's decomposition method for solving an intermediate fractional 
advection-dispersion equation," Computers \& Mathematics with Applications, vol. 59, no. 5, pp. 1759-1765, 2010.

[11] J. He, "A new approach to nonlinear partial differential equations," Communications in Nonlinear Science and Numerical Simulation, vol. 2, no. 4, pp. 230-235, 1997.

[12] G.-c. Wu and E. W. M. Lee, "Fractional variational iteration method and its application," Physics Letters A, vol. 374, no. 25, pp. 2506-2509, 2010.

[13] S. Guo and L. Mei, "The fractional variational iteration method using He's polynomials," Physics Letters A, vol. 375, no. 3, pp. 309-313, 2011.

[14] J.-H. He, "Homotopy perturbation technique," Computer Methods in Applied Mechanics and Engineering, vol. 178, no. 3-4, pp. 257-262, 1999.

[15] J.-H. He, "A coupling method of a homotopy technique and a perturbation technique for non-linear problems," International Journal of Non-Linear Mechanics, vol. 35, no. 1, pp. 37-43, 2000.

[16] Z. Odibat and S. Momani, "A generalized differential transform method for linear partial differential equations of fractional order," Applied Mathematics Letters of Rapid Publication, vol. 21, no. 2, pp. 194-199, 2008.

[17] M. Cui, "Compact finite difference method for the fractional diffusion equation," Journal of Computational Physics, vol. 228, no. 20, pp. 7792-7804, 2009.

[18] Q. Huang, G. Huang, and H. Zhan, "A finite element solution for the fractional advection-dispersion equation," Advances in Water Resources, vol. 31, no. 12, pp. 1578-1589, 2008.

[19] S. Guo, L. Mei, Y. Li, and Y. Sun, "The improved fractional subequation method and its applications to the space-time fractional differential equations in fluid mechanics," Physics Letters A, vol. 376, no. 4, pp. 407-411, 2012.

[20] B. Lu, "Bäcklund transformation of fractional Riccati equation and its applications to nonlinear fractional partial differential equations," Physics Letters A, vol. 376, no. 28-29, pp. 2045-2048, 2012.

[21] S. Zhang and H.-Q. Zhang, "Fractional sub-equation method and its applications to nonlinear fractional PDEs," Physics Letters A, vol. 375, no. 7, pp. 1069-1073, 2011.

[22] G. Jumarie, "Modified Riemann-Liouville derivative and fractional Taylor series of nondifferentiable functions further results," Computers \& Mathematics with Applications, vol. 51, no. 9-10, pp. 1367-1376, 2006. 


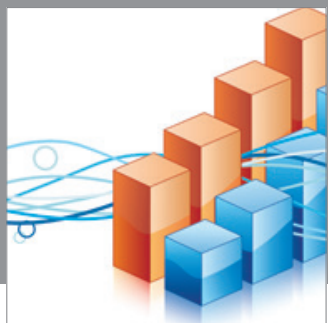

Advances in

Operations Research

mansans

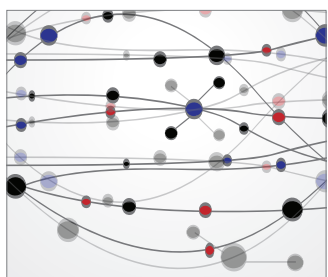

The Scientific World Journal
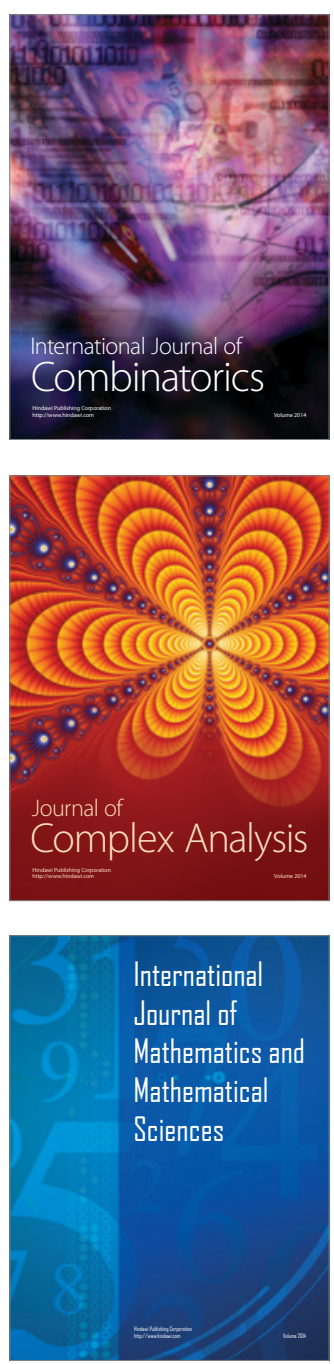
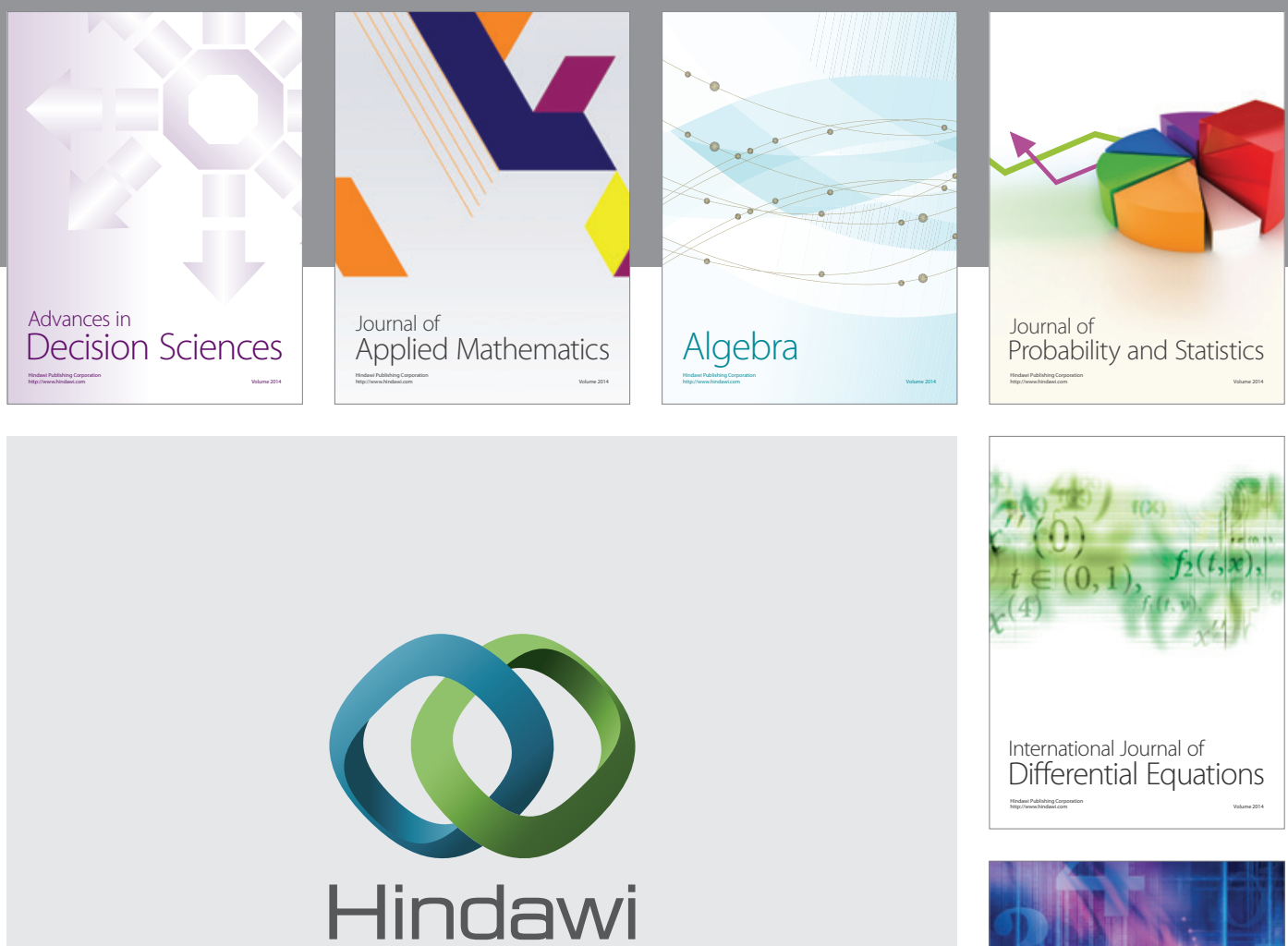

Submit your manuscripts at http://www.hindawi.com
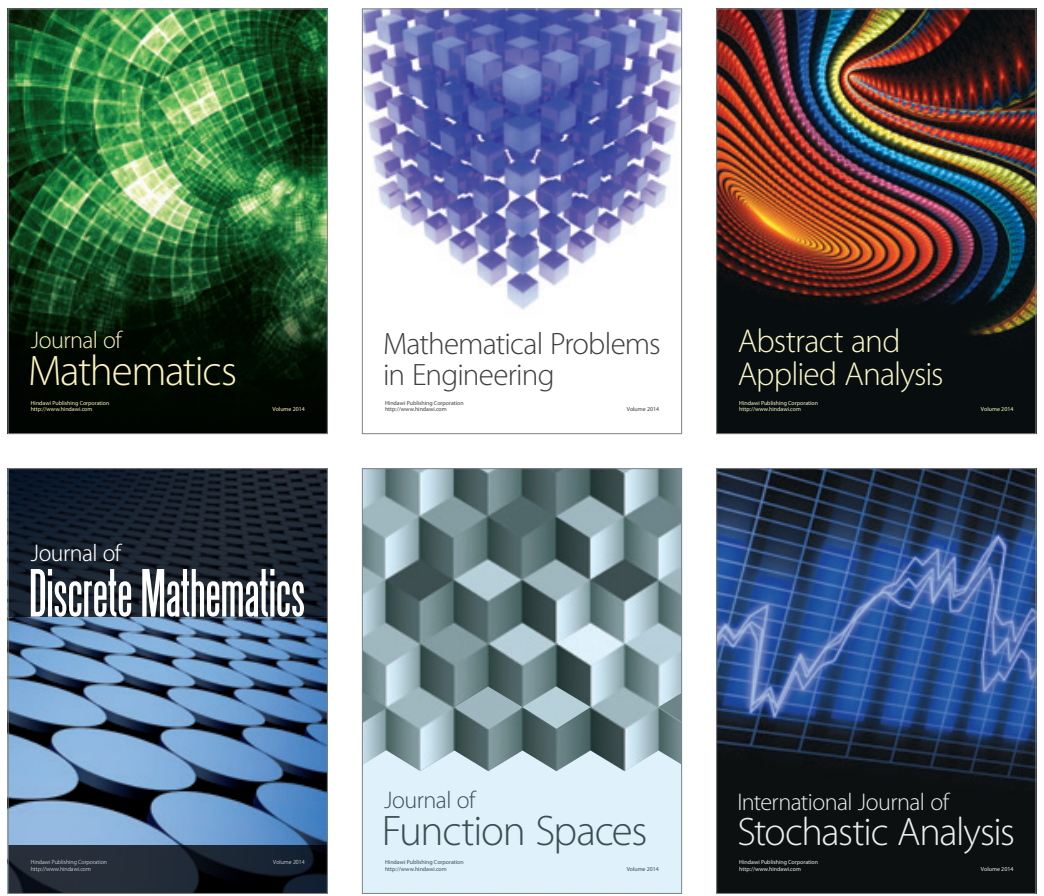

Journal of

Function Spaces

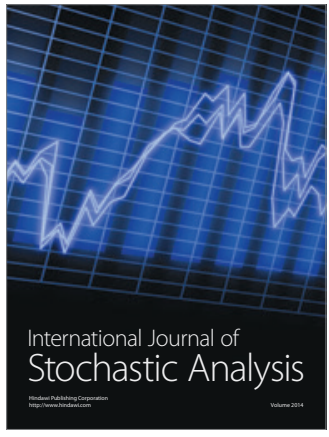

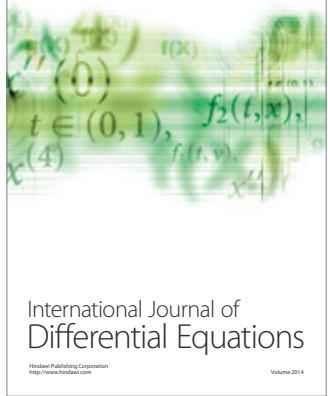
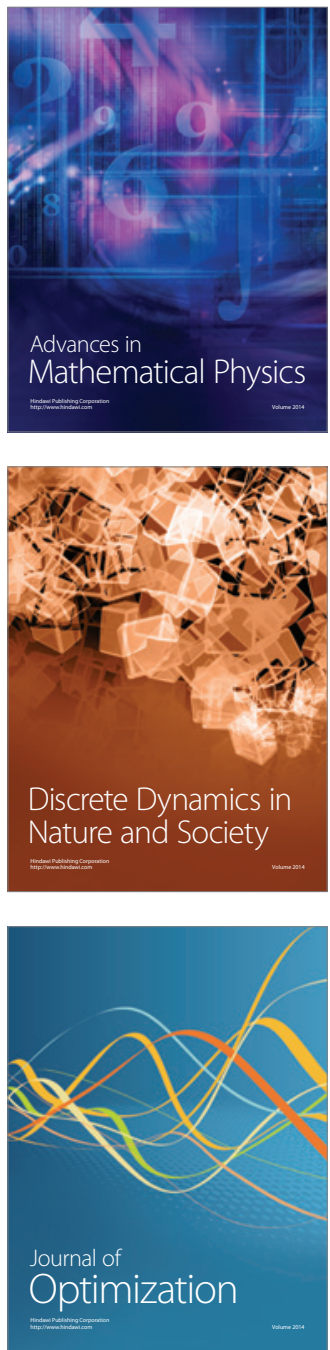